\title{
Research Protocol for a Before-After Comparison of the Impact of COVID-19 on Physical Activity, Sedentary and Dietary Behaviour of School-Going Adolescents in Punjab, India (ICPASA Study)
}

\section{Supriya Thapar ( $\sim$ supriya.thapar@maastrichtuniversity.nl )}

Post Graduate Institute of Medical Education and Research https://orcid.org/0000-0002-3023-505X

Maartje Willeboordse

Maastricht University Faculty of Health Medicine and Life Sciences: Universiteit Maastricht Faculty of Health Medicine and Life Sciences

\section{Onno CP van Schayck}

Maastricht University Faculty of Health Medicine and Life Sciences: Universiteit Maastricht Faculty of Health Medicine and Life Sciences

\section{Anita Vreugdenhil}

Maastricht University Medical Centre+: Maastricht Universitair Medisch Centrum+

Jarnail Singh Thakur

Post Graduate Institute of Medical Education and Research School of Public Health Department of Community Medicine

\section{Methodology}

Keywords: Adolescents, physical activity, sedentary and dietary behaviour, COVID-19

Posted Date: September 1st, 2021

DOl: https://doi.org/10.21203/rs.3.rs-840081/v1

License: (a) This work is licensed under a Creative Commons Attribution 4.0 International License. Read Full License

Version of Record: A version of this preprint was published at Advances in Nutrition \&amp; Food Science on March 14th, 2022. See the published version at https://doi.org/10.33140/ANFS.07.01.007. 


\section{Abstract}

Background: The COVID-19 pandemic has exacerbated the already existing pandemic of physical inactivity. In adolescents, the effect of COVID-19related governmental measures may beeven more pronounced,as they experience school closures, home confinement, social distancing and possible mental health issues as a result.The currentstudy uses the COVID-19 pandemic and associated governmental measures as a natural experiment to understand the impact it may have on the lifestyle of adolescents with relation to physical activity, sedentary and dietary behaviour. We also aim to describe the determinants of change in lifestyle behaviours.

Methods/Design: A before-after experimental study design will be followed in which baseline measurements have been undertaken in 2018-19 before the COVID-19 pandemic, and the follow-up measurements will be repeated during the COVID-19 pandemic. Twenty government and private schools of Punjab, India form the primary unit of participation from which 1086adolescents participated in the study. Validated questionnaires are used to measurephysical activity, sedentarybehaviour, and dietary behaviour. Additionally, objective measures of physical activity will be performed using ActiGraph wGT3X-BT over a 7-day period on a sub-sample. Body mass index (BMI) and waist circumference will be assessed objectively as a measure of central obesity. Qualitative interviews and focus group discussions will be conducted to have a better understanding for reasons of change in physical activity, sedentary and dietary behaviour or any possible differences between groups.

Discussion: The study aims torevealthe impact of COVID-19 governmental measures on physical activity, sedentary and dietary behaviour of adolescents. It is one of the first studies to show both subjective and objective measures of physical activity levels and sedentary time of school-going adolescents during COVID-19. Due to the mixed method approach we will be able to identify reasons for changes in lifestyle behaviours and possibly also detect innovative ways of stayingphysically active during the era of physical distancing.

\section{Introduction}

Adolescence marks a critical period of life as the development of risk factors and behaviour formation, such as patterns of activity in adulthood, are established during this stage $(1,2)$. World Health Organization recommends children and adolescents in the age group of 5-17 years to accumulate at least 60 minutes of moderate to vigorous physical activity per day, most of which should be aerobic ( 3 , 4). As of 2016, about $80 \%$ of school-going adolescents aged $11-17$ years perform insufficient physical activity (PA) with girls being less active that boys (5). Studies show that physical activity (PA) declines with age (6) and that adolescents who become lesser active with age are at a higher risk of becoming obese in adulthood than those who stay active (7). Physical inactivity, during childhood and adolescence also increases the risk of development of obesity related comorbidities such as hypertension, dyslipidemia, diabetes, and cardiovascular diseases. In 2018, WHO developed a Global Action Plan for 
Physical Activity to promote policy decisions on physical activity and provided new guidelines for a $15 \%$ reduction in physical inactivity by 2030 (8).

Following the outbreak of novel coronavirus disease (COVID-19) and its declaration as a pandemic in March 2020 by the World Health Organization, school closures have been an ubiquitous approach by governments to prevent community transmission. By June 2020, about 1 billion children and adolescents in 112 countries were affected by school closures (9). With the ongoing COVID-19 situation and associated school closures, the physical activity levels are expected to fall furthermore with a simultaneous increase in amount of time spent sedentary. Physical inactivity was declared a pandemic in 2012 (10), and current data suggests that it will persist long after we recover from the COVID-19 pandemic (11).

Home confinement and social distancing not only reduces regular physical activity (PA) in adolescents due to closure of schools and access to sports facilities, playgrounds and parks, but also exacerbates the lifestyle related risk factors of obesity such as unhealthy snacking and increase in sedentary behaviour (12-14). In a study conducted between 27-31 March 2020, South Korean parents reported an increase in screen time in $81 \%$ of the children and a decrease in the use of play or sports facility the physical activity levels in $94 \%$ of the children (15). In the Netherlands, the proportion of children meeting the WHO PA guidelines of $\geq 60 \mathrm{~min} /$ day of moderate to vigorous physical activity (MVPA) decreased from $64 \%$ before pandemic to $20 \%$ during the pandemic (16). In Verona, Italy, sports time of obese children decreased as a result of home confinement, while their sleep and sedentary time increased during the same time (13). Data from India is scant, especially for adolescents, however, a recent study reports a reduction in physical activity of $38.6 \%$ Indians between 13-25 years during the pandemic led lockdown (17). School closures are also expected to lead to the emergence of mental health issues due to extended confinement, and other youth health services, and access to school meals. Physical inactivity may lead to complications such as lack of sleep, low social interactions, and a further risk of cardiovascular diseases. The situation poses a greater challenge for adolescents as they develop independence and begin to prioritize connections with their friends and family during this time (18).

As children and adolescents are forced to stay at home and education takes place through various online platforms, increase in screen time is inevitable. Children of working parents, single parents, or parents working from home may have a higher accumulation of screen time. Education through virtual platforms reinforces the educational rights of individuals besides keeping them connected with their friends and peers even in the time of social distancing, thereby providing social support. Although, greater screen time (>2 hours/day) is associated with poor sleep, it is vital to utilize this opportunity for online physical activity classes, exercise videos and even social support services for mental health (19).

Apart from physical activity and sedentary behaviour, healthy eating habits are fundamental for good health during adolescence and for the prevention of developing obesity related noncommunicable diseases during adulthood. The pandemic led lockdown has demonstrated a rise in unhealthy eating habits among adolescents such as snacking between meals and eating out of control as demonstrated in 
a study conducted in European countries. This may be attributed to boredom and mood swings as a result of social distancing (20). It is therefore important to understand the effect of home confinement on dietary behaviour, especially when we are aware that a healthy diet is imperative for immunity against COVID-19 (21).

The WHO recognizes schools as one of the most promising settings for promotion of physical activity and other healthy lifestyle behaviours among children and adolescents (6). Having this in mind, the current study was initially designed as a cluster randomized trial, with 20 schools and a minimum enrolment of 834 adolescents, to test the effectiveness of a health promotion intervention on physical activity of school going adolescents in Punjab- PASA Study. It was approved by Institute Ethics Committee, Post Graduate Institute of Medical Education and Research (PGIMER), Chandigarh (NK/6692/Study/525) in January 2018 and registered in the Netherlands Trial Register (NL8309). Baseline measurements were completed in February 2019. However, COVID-19 pandemic and governmental measures instated in India in March 2020 led to school closures and the intervention could not start. The nation-wide lockdown continued till May 2020 and schools remain to be closed even after. Therefore, the above stated reasons combined with the social and environmental aspect of promotion of physical activity in schools, the research group of the study decided to change the aim of the research as per the current need. We want to assess the impact of governmental measures on physical activity, sedentary and dietary behaviour of adolescents, and how this relates to health parameters. Students are attending both online and offline classes during follow-up measurements due to COVID-19. We would like to measure a change independent of attending school, but dependent on COVID-19 related governmental measures as a whole.

Keeping in view these ideas, the objectives of the study have been redefined as (a) assess the impact of COVID-19 associated school closures on physical activity and sedentary behaviour of adolescents attending schools in Mohali (SAS Nagar) city, Punjab, (b) examine how the COVID-19 pandemic has affected the nutrition and health status of adolescents with respect to over-nutrition and childhood obesity (c) understand the determinants of lifestyle change due to the pandemic and what measures may be taken to improve the daily routine of adolescents keeping in mind physical distancing. We hypothesize that the school closures due to COVID-19 pandemic has led to a decrease in proportion of adolescents performing adequate physical activity according to WHO guidelines.

\section{Methodology}

\section{Setting and design}

The Impact of Covid-19 on Physical Activity, sedentary and dietary behaviour of School-going Adolescents (ICPASA) study will be carried out in schools of Mohali (SAS Nagar) city of Punjab state in the northern region of India and adopts a 'natural experiment' approach. The UK Medical Research Council (MRC) defines natural experiment as 'events, interventions or policies which are not under the control of researchers, but which are amenable to research which uses the variation in exposure that they 
generate to analyze their impact'(22). In this study, adolescents are exposed to COVID-19 related governmental measures of school closures, social distancing and home confinement which act as a natural experiment. Thus, the current study is a before-after experimental study design in which subjects are measured before the pandemic during baseline and exposed to COVID-19 as a natural experiment starting March 22, 2020. The baseline measurements were conducted in September 2018- February 2019, while follow-up measurements will be conducted in 2021. The study uses quantitative methods to measure the change in proportion of adolescents performing physical activity as per WHO guidelines, and their sedentary and dietary behaviours, before and during COVID-19 pandemic and associated school closures. The study also employs qualitative measures to understand the determinants for changes in lifestyle behaviours.

\section{Recruitment}

Following permission by the Secretary, School Education, Department of Education, Punjab in June 2018, a list of public and private schools in Mohali (SAS Nagar) district was obtained from them. All schools falling under the area of SAS Nagar Municipal Corporation were identified as eligible for inclusion. A total of 20 schools (10 government and 10 private schools) were randomly selected from the school list. The Department of Education, Punjab, sent a letter to the selected schools informing about the study following which the school heads were approached to discuss the study and give their consent. Since school support is necessary, participation of the schools was on voluntary basis. One of the ten private schools which were not willing to participate was randomly replaced with another school from the list. All school-going adolescents studying in schools of SAS Nagar (Mohali) city between the ages of 10-19 years were eligible for inclusion. The course of the study is illustrated in Fig. 1.

Students who were studying in sixth to eighth standard at the time of baseline data collection (September 2018 - February 2019) were selected from each participating school. Consent forms and information sheet were distributed to 1308 students who were asked to discuss with their families about participation in the study. Only those students who brought back signed consent forms from their parents/guardians were eligible for inclusion in the study and were asked to fill assent forms. About $83 \%$ students $(n=1086)$ submitted both consent and assent forms and participated in the baseline measurements. In middle schools which enroll students up to eighth standard, students from sixth standard were purposively selected in order to be able to repeat the measurements with the same subjects at the end of the study.

\section{Power Calculation}

The baseline proportion of students reaching PA levels of $\geq 60$ minutes/day in the present sample is $35.4 \%$. Therefore, 0.354 is taken as the true proportion of the population. Although studies have shown a steep decline in physical activity levels of children and adolescents during school closures as a result of the COVID-19 pandemic, the baseline PA levels in the current sample is quite low. Thereby, expecting a smaller decrease of $6.5 \%$ (14) in proportion of physically active adolescents, the null hypothesis 
proportion is assumed as 0.289 . Due to COVID-19 situation, resultant school closures, and limited access to mobile and internet, we are assuming a high drop-out of $45 \%$ in follow-up measurements as compared to baseline. Therefore, power calculation is done for $55 \%$ of 1086 adolescents, i.e. approximately 600 adolescents. If pre and post measurements are recorded for 600 adolescents, we have a power of about $91 \%$, which is a good power to observe the differences over time that occurred as a result of COVID-19.

\section{Study Outcome \\ Primary Outcome}

The change in proportion of adolescents reaching the recommended level of physical activity using selfreported measures as per WHO guidelines during COVID-19 will be the primary outcome of the study.

\section{Secondary Outcome}

Secondary outcomes of the study include lifestyle changes in adolescents in terms of physical activity and sedentary behaviours, and dietary modifications, using self-report measures; objective measures of physical activity (assessed in a subsample); changes in health parameters, particularly BMI and waist circumference will be assessed as indicators of undernutrition and overweight/obesity. The determinants of change in lifestyle behaviours of adolescents as described during the qualitative interviews and focus group discussions (FGDs) are also a secondary outcome.

\section{Data Collection Tools For Baseline Measurements}

\section{Measurement of physical activity, sedentary and dietary behaviours}

The study adopts a 'natural experiment' approach in which baseline measurements were done for the study planned earlier, while follow-up measurements will be done while the governmental measures to prevent the spread of COVID-19, such as social distancing and online schooling are still in place. Baseline measurements included quantitative assessment of physical activity and dietary behaviour using a modified version of GSHS-2006 questionnaire. The Physical Activity Module of the Global School based Student Health Survey (GSHS) Questionnaire 2006 is used as a subjective measure for the physical activity and sedentary behaviour in school-going adolescents. The physical activity module was modified to meet the objectives of the study. Questions for leisure time physical activity were included from the BAECKE questionnaire (23). Questions for recess time PA were added from PAQ-C questionnaire (24). Dietary behaviours module of the GSHS 2006 questionnaire is used as it is. The questionnaire was pretested in a similar population by self-administration method. It was translated and back translated into vernacular language (Punjabi) and pilot tested. 


\section{Objective Measure Of Physical Activity By Accelerometry}

The ActiGraph wGT3X-BT, which is a triaxial accelerometer, is used to objectively measure the physical activity of adolescents. Schools were chosen based on convenience sampling as only seven schools who agreed that adolescents wear accelerometers around their waist for seven days were given the ActiGraphs. The selection of students wearing the ActiGraph was also on voluntary basis. A sub-group of students $(n=233$ ) were given verbal instructions to wear ActiGraphs for 7 days mounted on the right side of the waist in front of the right hip at all times except during sleeping and performing water activities such as bathing, swimming etc. Efforts were made to equally distribute the ActiGraph among government and private schools. The Actigraphs were distributed to the adolescents one school after another, by initializing for date and time using the ActiLife6 software. The raw acceleration data will be converted into objective activity with the help of ActiLife6. Outcome measures of interest are duration of sedentary

time, light physical activity, moderate to vigorous physical activity (MVPA), metabolic equivalents (METs), average step count, and counts per minute (CPM).

\section{Anthropometry Measurements}

Objective anthropometric measurements were performed on study participants. A flat surface inside the classroom or infirmary of the school was chosen for weight and height measurements. Weight of the adolescents was measured in kilograms up to one decimal point using Seca 813 digital flat scale. Students were asked to be in light clothing i.e. remove sweaters/blazers, belts, and shoes before taking measurements. Height was measured in centimeters using Seca 213 portable stadiometer. Light clothing and being barefoot will be followed for height measurement as well. Students were instructed on the correct posture for height measurement; to touch the heels to the back of the stadiometer wall, legs positioned straight, shoulders relaxed and head looking straight ahead. The research staff was trained to note the height of the pupil avoiding any parallax. Waist circumference was measured in centimeters using the Seca 203 measuring tape. Research staff explained the procedure to the adolescents and asked them if they are comfortable in lowering their pants/skirts for the measurement. Upon their consent, waist circumference was measured individually with each adolescent standing in a relaxed position and breathing normally. Measurement was done on the right side of the pupil after they exhale. Any parallax was avoided. In case of uncertainty in any of the measurements, the result was recorded three times and the average will be taken as the final measurement. Body Mass Index (BMI) of adolescents will be calculated using individual measures of weight and height and manually applying the formula for calculation.

\section{Interviews}

Qualitative data was collected in the form of focus group discussions (FGDs) and semi-structured interviews with the participants of the study, that is, the adolescents, school staff, and parents. In this 
study, FGDs were held with the adolescents, their parents and school staff after completion of baseline measurements to aid in better understanding of the determinants of physical activity and sedentary behaviour among adolescents.

\section{Data Collection During Covid-19}

Follow-up measurements will be conducted with the same population as baseline in the form of online questionnaires, and anthropometric measurements will follow as schools start to reopen with the recommended COVID-19 restrictions. Schools that participated during baseline will be invited for followup measurements. All measurements conducted during baseline, will be repeated following the same protocol, with some adjustments. The questionnaire will be web-based and schools will be asked to share the web-link of questionnaire on their preferred platform of online information sharing. Schools can choose whether they prefer web-based or paper-based questionnaires. The GSHS-2006 questionnaire is modified to include questions on lifestyle changes in adolescents due to COVID-19. It is created on Google Forms in both English and Punjabi language and has been pre-tested on a similar population before roll-out in the study participants. Students can choose their preferred language of the survey. Physical activity will be objectively measured using ActiGraph over a 7-day period in the same subsample. Sanitization of the accelerometers will be done after each use. All anthropometric measurements (weight, height and waist circumference) will be repeated in follow-up using the same procedure as in baseline measurements. The latest guidelines issued by the government to prevent the spread of COVID19 will be followed. Focus group discussions and interviews will be conducted with students, parents and school staff from the sample population, but not the same as that during baseline, to supplement quantitative data and understand the reason for changes in lifestyle behaviour of adolescents that may have arisen, but are not limited to socioeconomic status, school type and curriculum. It will also aim to discover innovative approaches of staying active during the COVID-19 era of physical distancing.

\section{Data Analysis}

The causal inferences of a natural experiment of COVID-19 related governmental measures of school closures, social distancing and home confinement will be derived related to physical activity and lifestyle changes in adolescents. Data will be analyzed using SPSS software version 26. Data will be tested for normality using the Shapiro-Wilk test. Before-after changes in physical activity levels, sedentary and dietary behaviours of adolescents will be analyzed using paired t-tests for continuous data, and the McNemar tests for categorical data or proportions. Qualitative data will be analyzed using thematic analysis.

\section{Discussion}

The current study aims to assess the impact of COVID-19 related governmental measures on the physical activity, sedentary behaviour and dietary behaviour of adolescents. The study is unique for the collection 
of objective measurement of physical activity of adolescents using accelerometers which has rarely been done in this population before, even more so during COVID-19. The study also assesses health parameters such as BMI and waist circumference before and during the pandemic enabling the evaluation of correlations between lifestyle changes and health and obesity of adolescents during the pandemic. One of the novelties of the study is that it focuses on the lifestyle of adolescents during the COVID-19 pandemic, adolescence being the habit forming age. Therefore, finding ways to keep the adolescents active in the era of physical distancing will go a long way in building pandemic-proof habits to stay healthy and also prevent obesity. Using this mixed-methods approach in which quantitative and qualitative data complement each other, we will also be able to explain the objectively measured effects.

A limitation of the study is that due to COVID-19 situation, the entire population may be difficult to reach for follow-up measurements. Only a sub-group of students who are attending schools will be available for objective measurement of physical activity via ActiGraph, and for anthropometric measurements, whereas the entire population from baseline measurements will be reached out for subjective measurements via online questionnaire. However, as shown in the power calculation, even with data from $55 \%$ percent of the students participating at baseline $(n=600)$ the current design has a good power to prove the study results.

\section{Conclusion}

The governmental measures of home confinement and social distancing will be taken as a natural experiment to derive insights on how school closures may have an impact on physical activity and lifestyle of adolescents. Results from the study may contribute to a global comparison of the effects of governmental measures on the lifestyle of adolescents. Findings from the study will help to develop recommendations for school-based physical activity interventions and assist in preparedness for the future, especially when physical distancing measures may still have to be in place even after schools reopen.

\section{Declarations}

- Ethics approval and consent to participate:The project was submitted and approved (NK/4026/Study) by Institute Ethics Committee, Post Graduate Institute of Medical Education and Research (PGIMER), Chandigarh, as on 17.01.2018. It was resubmitted with the aforementioned modifications due to COVID-19 and approved on 5.11.2020 as NK/6692/Study/525. The study is also registered in the Netherlands Trial Register (NL8309). Informed consent from parents and assent from children was received after explaining the study purpose in their own language. Informed consent will be taken for recording the in-depth interviews and focus group discussions. Confidentiality will be ensured by removing the personal identifiers from the data set.

- Consent for publication: Not Applicable because this manuscript does not contain any individual person's data in any form. 
- Availability of data and materials: The data are not yet available.

- Competing interests: The authors declare that they have no competing interests.

- Funding:There is no funding for the study.

- Authors' contributions: All authors were involved in designing of the study. ST drafted the manuscript and was involved in participant recruitment. JST initiated the project and received ethics approval. $S T, M W, C v S$ and $A V$ were involved in editing and refining the arguments presented in the final manuscript. All authors critically reviewed the final version of the manuscript.

- Acknowledgements: The authors would like to express a big thanks to Department of Education, Punjab, for extending their support in carrying out the study. We would also like to thank the Principals, teachers and staff of all the participating schools who helped with the arrangements to make it happen. We have immense gratitude for the adolescents who participated in this study and their families, who supported them.

- Authors' information (optional): None

\section{References}

1. Gråstén A, Yli-Piipari S, Watt A, Jaakkola T, Liukkonen J. Effectiveness of School-Initiated Physical Activity Program on Secondary School Students' Physical Activity Participation. Journal of School Health.85(February 2015).

2. Sutherland R, Reeves P, Campbell E, Lubans DR, Morgan PJ, Nathan N, et al. Cost effectiveness of a multi-component school-based physical activity intervention targeting adolescents: the 'Physical Activity 4 Everyone'cluster randomized trial. International Journal of Behavioural Nutrition and Physical Activity. 2016 Dec;13(1):1-4.

3. WHO W. Global recommendations on physical activity for health. Geneva World Heal Organ. 2010 Apr;60.

4. Chaput JP, Willumsen J, Bull F, Chou R, Ekelund U, Firth J, et al. 2020 WHO guidelines on physical activity and sedentary behaviour for children and adolescents aged 5-17 years: summary of the evidence. International Journal of Behavioural Nutrition and Physical Activity. 2020;17(1):1-9.

5. Guthold R, Stevens GA, Riley LM, Bull FC. Global trends in insufficient physical activity among adolescents: a pooled analysis of 298 population-based surveys with 1.6 million participants. The Lancet Child \& Adolescent Health. 2020 Dec;17(1):1-9.

6. Dobbins M, Husson H, DeCorby K, LaRocca RL. School-based physical activity programs for promoting physical activity and fitness in children and adolescents aged 6 to 18 . Cochrane database of systematic reviews. 2013(2).

7. Kwon S, Janz KF, Letuchy EM, Burns TL, Levy SM. Active lifestyle in childhood and adolescence prevents obesity development in young adulthood. Obesity. 2015 Dec;23(12):2462-9.

8. World Health Organization. Global action plan on physical activity 2018-2030: more active people for a healthier world. World Health Organization; 2019 Jan 21. 
9. Global Education Coalition Paris, France: UNESCO; 2020 Available from:

https://en.unesco.org/covid19/educationresponse/globalcoalition. Accessed 9 June 2020

10. Lancet Physical Activity Series Working Group. The pandemic of physical inactivity: global action for public health. The lancet. 2012 Jul 21;380(9838):294-305.

11. Hall G, Laddu DR, Phillips SA, Lavie CJ, Arena R. A tale of two pandemics: How will COVID-19 and global trends in physical inactivity and sedentary behaviour affect one another? Prog Cardiovasc Dis. 2021 Jan;64:108

12. Di Renzo L, Gualtieri P, Pivari F, Soldati L, Attina A, Cinelli G, et al. Eating habits and lifestyle changes during COVID-19 lockdown: an Italian survey. J Transl Med. 2020 Dec;18:1-5.

13. Pietrobelli A, Pecoraro L, Ferruzzi A, Heo M, Faith M, Zoller T, et al. Effects of COVID-19 Lockdown on Lifestyle Behaviours in Children with Obesity Living in Verona, Italy: A Longitudinal Study. Obesity. 2020 Aug;28(8):1382-5.

14. Ruiz-Roso MB, de Carvalho Padilha P, Matilla-Escalante DC, Brun P, Ulloa N, Acevedo-Correa D, et al. Changes of Physical Activity and Ultra-Processed Food Consumption in Adolescents from Different Countries during Covid-19 Pandemic: An Observational Study. Nutrients. 2020 Aug;12(8):2289.

15. Guan H, Okely AD, Aguilar-Farias N, del Pozo Cruz B, Draper CE, El Hamdouchi A, et al. Promoting healthy movement behaviours among children during the COVID-19 pandemic. The Lancet Child \& Adolescent Health. 2020 Jun 1;4(6):416-8.

16. Ten Velde G LJ, Arayess L, van Loo C, Hesselink M, Reijnders D, Vreugdenhil A. Physical activity behaviour and screen time in Dutch children during the COVID-19 pandemic: Pre-, during- and postschool closures. Pediatr Obes. 2021; Feb 23:e12779.

17. Roy S. TS, Kanchan S., Bajpai P. Impact of COVID-19 pandemic led lockdown on the lifestyle of adolescents and young adults. medRxiv preprint. 2020 Jan 1.

18. Lancet Child Adolescent Health. Pandemic school closures: risks and opportunities. Lancet Child Adolesc Health. 2020 May;4(5):341.

19. Nagata JM, Abdel Magid HS, Pettee Gabriel KP. Screen Time for Children and Adolescents During the Coronavirus Disease 2019 Pandemic. Obesity (Silver Spring, Md.). 2020 May 28.

20. Ammar A, Brach M, Trabelsi K, Chtourou H, Boukhris O, Masmoudi L, et al. Effects of COVID-19 Home Confinement on Eating Behaviour and Physical Activity: Results of the ECLB-COVID19 International Online Survey. MedRxiv. 2020 Jan 1.

21. Self Help for Good Health: A Naturopathy Perspective. Government of India. Available from: https://blog.mygov.in/self-help-for-good-health-a-naturopathy-perspective/ . 2020; Sep 03

22. Using natural experiments to evaluate population health interventions. Glasgow: Medical Research Council. 2011.

23. Pols MA, Peeters PH, Bueno-de-Mesquita HB, Ocke MC, Wentink CA, Kemper HC, et al. Validity and repeatability of a modified Baecke questionnaire on physical activity. International journal of epidemiology. 1995 Apr 1;24(2):381-8. 
24. Kowalski KC, Crocker PR, Donen RM. The physical activity questionnaire for older children (PAQ-C) and adolescents (PAQ-A) manual. College of Kinesiology, University of Saskatchewan. 2004 Aug;87(1):1-38.

\section{Figures}

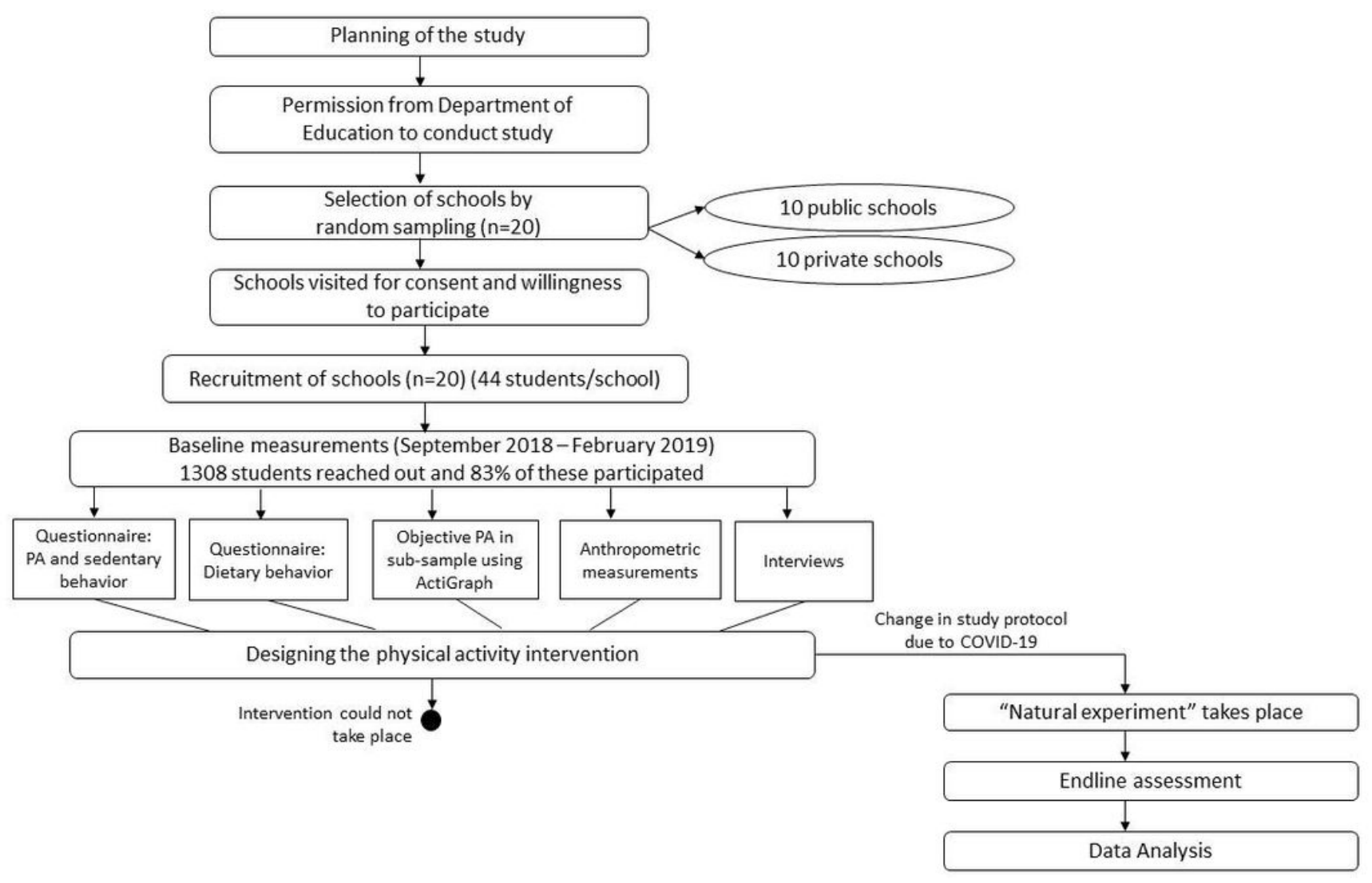

Figure 1

Flowchart of the study 